\title{
IQWiG lässt Hypoglykämiegefahr außer Acht
}

\author{
Die Deutsche Diabetes-Gesellschaft (DDG) befürchtet Therapieeinschränkungen für Diabetespatien- \\ ten in Deutschland, nachdem sowohl für Linagliptin als auch Dapagliflozin vom IQWiG kein Zusatz- \\ nutzen gegenüber Sulfonylharnstoffen bescheinigt wurde. Und sie protestiert heftig.
}

Der Gemeinsame Bundesausschuss (G-BA) hatte am 21. Februar 2013 entschieden, dass der DPP-4-Hemmer Linagliptin keinen Zusatznutzen gegenüber Sulfonylharnstoffen $(\mathrm{SH})$ bietet. Die DDG hält diese Entscheidung für wissenschaftlich nicht nachvollziehbar und sieht die Therapiesicherheit von Hunderttausenden in Deutschland gefährdet. Sollten politische Entscheidungen Medikamente wie den DPP-4-Hemmer vom deutschen Markt verdrängen, befürchtet die DDG gravierende therapeutische Einbußen für rund 650000 Menschen mit Diabetes mellitus. „Als Konsequenz dieses Beschlusses müssten Tausende Diabetespatienten auf die Vorteile der DPP-4-Inhibitoren verzichten und auf Sulfonylharnstoffe umgestellt werden“, so Prof. Stephan Matthaei, Präsident der DDG. Damit steige bei diesen Patienten die Gefahr zur Entwicklung von Hypoglykämien, die lebensbedrohlich werden können. Das sei für Betroffene und Ärzte rechtlich wie ethisch völlig inakzeptabel. Wie alle anderen internationalen wissenschaftlichen Fachgesellschaften auch, hält es die DDG aufgrund der vorliegenden Studien unzweifelhaft für erwiesen, dass Hypoglykämien mit DPP4 -Hemmern viel seltener auftreten als mit SH. „Für viele Patienten ist die Einnahme von DPP-4-Inhibitoren deshalb von Vorteil“, betont Prof. Andreas Fritsche, der DDG-Pressesprecher. Die europäische sowie die Amerikanische Diabetesgesellschaft (EASD, ADA) befürworten in einem gemeinsamen Positionspapier den Einsatz von DPP-4-Hemmern, wenn Hypoglykämien vermieden werden sollen. Dies betrifft besonders Patienten mit eingeschränkter Nierenfunktion, Herz- Kreislauf-Erkrankungen und ältere Patienten. Es gilt auch für Menschen mit Diabetes, bei denen schwere Unterzuckerungen berufsbedingt eine Eigen- oder Fremdgefährdung darstellen, etwa bei Berufskraftfahrern. Zumindest für diese Patienten hätte der G-BA einen Zusatznutzen für DPP-4-Hemmer attestieren müssen, so die DDG.

Der G-BA hatte die selteneren Unterzuckerungen mit Linagliptin nicht auf die Eigenschaften des Wirkstoffs, sondern auf unterschiedliche Therapiestrategien in den jeweiligen Studien zurückgeführt. Diese Einschätzung wies die DDG als unwissenschaftlich zurück - mit dem Hinweis, dass die Methodenkritik des G-BA widersprüchlich sei, wissenschaftlichen Standards widerspreche und ethisch nicht zu vertretende Vergleichsstudien fordere. Der Hersteller hatte Linagliptin in Deutschland noch vor der Marktzulassung zurückgezogen, da die negative Entscheidung des G-BA zu befürchten war.

Nun drohe in Deutschland die Nicht-Verfügbarkeit für die DPP-4-Hemmer, so die DDG. Da die Studien-Evidenzlage zu den anderen DPP-4-Hemmern (Sita-, Vilda- und Saxagliptin) der von Linagliptin sehr ähnlich ist, wird ihnen das Institut für Qualität und Wirtschaftlichkeit im Gesundheitswesen (IQWiG) (vermutlich Anfang 7/2013) ebenfalls keinen Zusatznutzen attestieren und der G-BA das Aus für die Wirkstoffklasse im August 2013 beschließen. „Dies würde die Patienten in Deutschland vom welt- weiten leitliniengerechten Therapiefortschritt komplett abkoppeln“, so Fritsche. Auch könnte Deutschland als Standort für wissenschaftliche und klinische Weiterentwicklungen an Attraktivität einbüßen. Linagliptin ist in Deutschland entwickelt worden und wird hier auch für den Weltmarkt produziert.

\section{Erneut vorrangig formale Kriterien herangezogen}

Das IQWiG hat auch einem Medikament der neuen Substanzklasse der SGLT-2-Hemmer den Zusatznutzen abgesprochen. Da zu erwarten ist, dass sich der G-BA am 23. April 2013 diesem Votum anschließt, wird diese effektive, sichere Therapie Typ-2Diabetikern in Deutschland nicht mehr angeboten werden können. Die DDG hält das IQWiG-Urteil aus wissenschaftlicher und medizinischer Sicht für falsch. Grund: Durch Hypoglykämien gefährdete Patienten profitieren von Dapagliflozin, dem ersten zugelassenen Medikament dieser Substanzklasse. Das IQWiG nehme mit seiner Entscheidung Hypoglykämien in Kauf, die tödlich sein können, kritisiert die DDG. Dies sei untragbar.

Der Sodium-Glukose Transporter 2 (SGLT-2) bewirkt in den Nieren die Wiederaufnahme von Glukose aus dem Urin. SGLT2-Hemmer unterbinden dies, sodass der Körper über den Harn vermehrt Glukose ausscheidet. Dies senkt Blutzucker, Blutdruck und Körpergewicht - eine günstige Kombination, die von keinem der bisher verfügbaren oralen Antidiabetika erzielt wird. Patienten nehmen die Tabletten kombiniert mit anderen Antidiabetika ein oder in Monotherapie. „Die neue Klasse der SGLT2-Inhibitoren schließt eine Lücke in der Therapie“, so Matthaei.

Die DDG kritisiert, dass das IQWiG erneut v.a. formale Kriterien heranzieht, um einem Medikament den Zusatznutzen abzusprechen und erneut die Hypoglykämiegefahr außer Acht lässt. Denn gegenüber SH verringert Dapagliflozin das Risiko von Hypoglykämien und ihrer potenziell lebensbedrohlichen Komplikationen. Der Patient nimmt das Medikament als sichere Standard-Tablette ein. „Die Sicherheit von Arzneimitteln ist aus unserer Sicht eindeutig ein erheblicher Zusatznutzen“, betonte Matthaei. In den vergangenen zehn Jahren nahmen Notaufnahmen vermehrt Menschen mit Typ-2-Diabetes und schweren Hypoglykämien auf. Dies geht u.a. auf die SH-Therapie zurück. Mit einer Absage an Präparate wie Dapagliflozin nehme das IQWiG potenziell tödliche Komplikationen in Kauf und behindere die Entwicklung in der Diabetologie, betonte Matthaei.

Wichtige Studien habe das IQWiG in seinem Urteil nicht berücksichtigt, kritisiert die DDG. Zudem ignoriere das Institut Vorgaben vieler internationaler Organisationen wie der EMA, die Dapagliflozin eine breite Zulassung erteilte. „Das IQWiG agiert in einer Weise, die normprägend sein könnte“, warnte Matthaei. Hier sei zu prüfen, ob das verfassungskonform ist. red Die vollständigen Stellungnahmen online unter: http://www.deutsche-diabetes-gesellschaft.de/stellungnahmen Quellen: Mitteilungen der DDG vom 27.2.2013 und 22.3.2013 